\title{
Investigação da Atenção do Estudante com técnicas de EEG: o uso da Realidade Virtual no Ensino
}

\author{
Andreia Solange Bos ${ }^{1,2}$, Milton Antônio Zaro' ${ }^{1}$, Michelle Câmara Pizzato ${ }^{2}$ \\ ${ }^{1}$ Universidade Federal do Rio Grande do Sul (UFRGS) \\ Porto Alegre - RS - Brazil \\ ${ }^{2}$ Instituto Federal do Rio Grande do Sul (IFRS) \\ Porto Alegre - RS - Brazil \\ \{andreia.bos@gmail.com, zaro@ufrgs.br,michelle.pizzato@poa.ifrs.edu.br
}

\section{Introdução}

Envolver os estudantes na aprendizagem é um princípio básico do ensino de graduação eficaz. Os resultados dos alunos envolvidos incluem experiências de aprendizagem significativas e habilidades aprimoradas em todos os domínios de aprendizagem. Neste estudo analisamos a influência da atenção de estudantes em diferentes formas de aprendizado ativo sobre o desenvolvimento de habilidades cognitivas, psicomotoras e afetivas. O acesso a internet nas salas de aula permite o aumento do uso da realidade virtual no ambiente escolar. A realidade virtual é um meio que permite às pessoas simularem o mundo real através de modelos $3 \mathrm{D}$ e interagir com avatares inteligentes. A interação é semelhante com um cenário do mundo real e portanto os professores podem usar a plataforma para fornecer experiências de aprendizado enriquecedoras para seus estudantes, (Bos, 2015). O OpenSim é uma plataforma que permite que as pessoas criem e personalize facilmente ambientes 3D que atendam às necessidades educacionais. Seguindo a mesma lógica, os museus virtuais oferecem aos visitantes virtuais a liberdade de interagir com um diálogo construtivo. (Bos, 2015). No trabalho de Bos et al, 2019b, é realizado um estudo sobre os estados de atenção do estudante com o uso de vídeos interativos utilizando um framework aberto como forma de investigar os tipos de atenção, e as mensurações encontradas mostram o estado mais efetivo do aluno. A seguir são abordadas as tecnologias utilizadas no estudo.

\section{Tecnologias}

\subsection{OpenSimulator}

OpenSim; abreviação de OpenSimulator - é um software livre e de código aberto que permite a qualquer pessoa criar um mundo virtual e rodar em seu próprio computador. $\mathrm{O}$ OpenSimulator é um servidor de aplicativos 3D de código aberto multi-plataforma e multiusuário.

\subsection{Mindwave NeuroSky}

O headset NeuroSky, utiliza um único sensor preso à testa do lado de fora do córtex cerebral no lobo frontal do cérebro, encarregado pelo nível de atenção e tarefas de memória de curto prazo (Neurosky, 2019). Assim, ele é utilizado para extrair os níveis de atenção e meditação do usuário, a partir da coleta de biossinais (ondas cerebrais) por eletrodos secos, (Bos et al, 2019a). Na figura 1 é mostrada o headset mindwave da neurosky. 


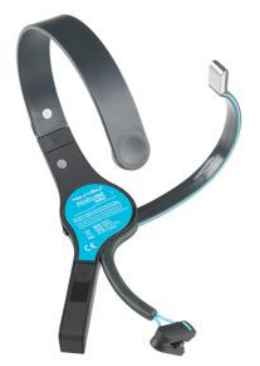

Figura 1. Neurosky Mindwave Fonte: Neurosky, 2019

Tais dispositivos são alternativas acessíveis para a monitoração da atividade cerebral de estudantes, pois têm a capacidade de medir ondas cerebrais referente a atenção e engajamento de forma não invasiva, (Bos et al, 2019a). Nesse sentido, o uso de sensores EEG portáteis na verificação da atenção de estudantes ao longo de atividades com realidade virtual pode ser uma excelente ferramenta de pesquisa.

\section{Métodos}

Para a implementação do Museu foram utilizados Scripts para tornar os artefatos interativos e o ambiente segmentado, aumentando assim a atenção do estudante. Na figura 2 é mostrada a imagem da confecção dos objetos educacionais com realidade virtual.

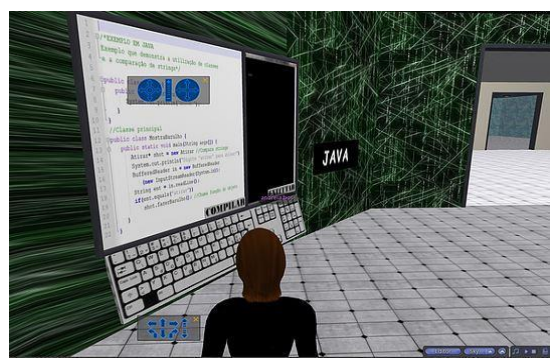

Figura 2. Confecção dos objetos em Realidade Virtual

\section{Metodologia}

Os estudantes foram convidados para realizar os estudos. Sendo que o Estudo 1 consistiu em o estudante realizar a leitura de um texto sobre introdução a computação. No estudo 2 é investigado a utilização com o Museu Virtual sobre o conteúdo de introdução à computação. A primeira parte da pesquisa foi realizada com o uso do sensor para mapear as ondas cerebrais dos estudantes durante a leitura de um texto, em que o aluno somente utilizava o sensorial visual para realizar. A segunda parte do estudo consistiu em fazer o uso da Realidade Virtual acessando o conteúdo que aborda a introdução a computação.

\subsection{Participantes}

Um total de sete voluntários (homens e mulheres, com idades entre 18 e 40 anos), principalmente de graduação da Universidade Federal do Rio Grande do Sul participaram do experimento, realizado no campus da universidade. Todos os participantes relataram terem um conhecimento básico de introdução a computadores. Após a visita foi colhido os biosinais disponibilizados como dados brutos no aplicativo do sensor.

\section{Resultados e discussão}


Os resultados do estudo foram de acordo com o início das expectativas. Propomos duas hipóteses examinando o efeito de atenção com texto e palavras, e o uso da plataforma de realidade virtual, investigando tipos de atenção e suas métricas nos apresentando perspectivas atualizadas. De acordo com os resultados alcançados em nosso estudo, a atenção afeta a recordação de conhecimentos prévios, isto indica que os professores devem considerar o esforço de atenção quando ocorre o ensino e principalmente a parte introdutória do conteúdo abordado. Na observação direta a análise de dados das métricas dos alunos mostram a importância sobre a estrutura do espaço virtual, do ambiente de aprendizagem, sua experiência exploratória e como foram abordados. A análise comparativa do estudo 1 e estudo 2 possibilitaram verificar que os alunos ao interagirem com o estudo 2 estavam mais efetivos em sua aprendizagem e estímulos de atenção.
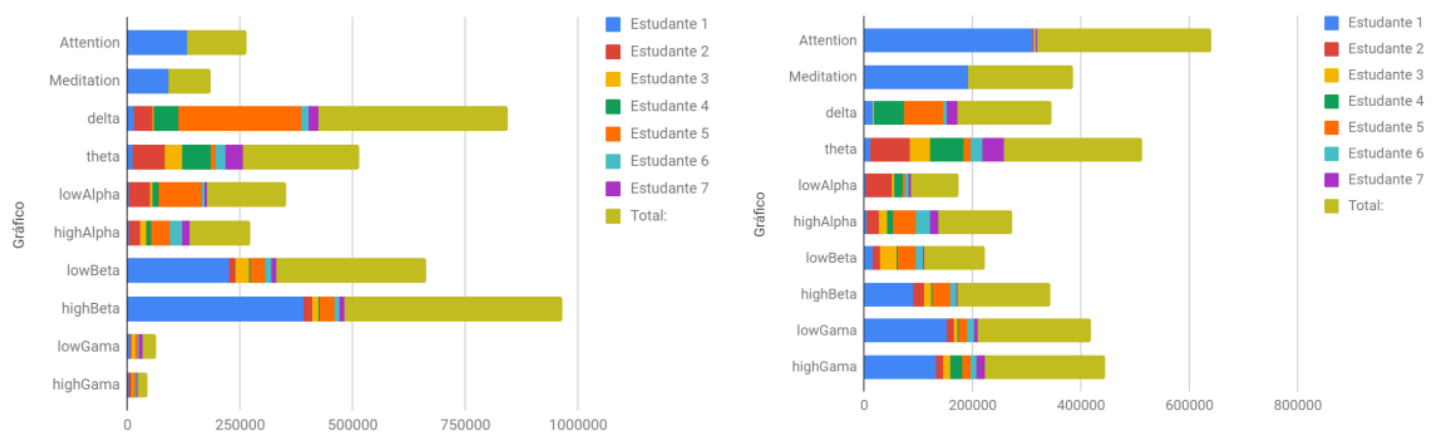

Gráfico 1. Variável considerada estudo 1 Gráfico 2. Variável considerada estudo 2

Como observado no gráfico 1 pode-se mensurar que os testes realizados com a leitura de texto, os sete estudantes estavam com diferentes estímulos neurais, natural do ser humano. Durante o estudo, as ondas de eletroencefalo mais estimuladas foram as que pontuaram em atenção, seguido da onda highBeta, ondas delta, ondas low Beta, ondas theta, low Alpha, high Beta, e as que menos mensuraram foram a high Gama e low Gama. Diante disso pode-se aferir que os estudantes estiveram mais tempo com as ondas neurais no córtex frontal com atenção, porém não tendo tanto estímulos visuais, espaciais e outros mais cognitivos, estavam e continuaram no caminho da menor resistência para o cérebro.

No gráfico 2 é possível mensurar que o estudo 2, com a utilização do museu de realidade virtual, os estudantes tiveram oscilações neurais diferenciadas em relação ao estudo 1. Durante o estudo 2 as ondas de eletroencefalo detectadas através dos feedback dos biosinais foram impulsionadas no córtex frontal que é onde se encontram as métricas de atenção do humano, seguida da frequência theta, high Gama, low Gama, meditação, delta, high Beta, high Alpha, low Beta e low Alpha. Sendo a frequência que menos oscilou foram as frequências low Alpha. A frequência high Gama é uma frequência que trabalha em 25 a $100 \mathrm{~Hz}$, sua frequência é extremamente rápida, uma onda que está no hipotálamo e que se move para frente em alta velocidade. Essa onda é relacionada quando é utilizada em alto processamento cognitivo para registrar novas informações nos sentidos e percepções. Os estados de atenção evidenciam picos desse tipo de onda neural. 


\section{Conclusões}

O estado de atenção do aluno na aprendizagem leva a uma compreensão mais profunda dos conceitos, ao aprimoramento do pensamento crítico e a resolução de problemas a níveis mais altos de desenvolvimento intelectual e a metacognição, (Bos et al, 2019a). Além disso o envolvimento e o estado de atenção do aluno ajuda a adquirir a experiência real, aprimorando suas habilidades sociais e interpessoais, melhorando as atitudes em relação ao aprendizado e a disciplina acadêmica. Concluímos também que a implementação de tarefas exploratórias em diversos contextos, se torna mais clara, mais atraente e útil. Eles se sentiram desafiados e motivados pelas tarefas e trabalharam com prazer. Este aspecto não só os ajudou a entender melhor o conteúdo, mas também facilito u o surgimento de formas mais criativas, coletivas e indivíduos que crescem com interações virtuais. Os alunos estimulam diferentes tipos de atenção para interagir e encontrar soluções, às vezes únicas e com ricos detalhes.

Agradecimentos: Ao IFRS (Instituto Federal do Rio Grande do Sul) e a UFRGS (Universidade Federal do Rio Grande do Sul).

\section{Referências}

Ausubel, D. P., Novak, J. D., \& Hanesian, H. (1968) "Educational Psychology: A Cognitive View"

Bos, A. S. (2015) "A.G.I.M.C: Agente Inteligente Conversacional Como Guia Em Um Museu Virtual 3D da Computação". Dissertação (Mestrado em Ciência da Computação) - PPGI, UFSM. Santa Maria.

Bos, A.; Zaro, M.; Prestes, L. P.; Pizzato, M. C.; Azevedo, D. F. G.;Avila, F. R.; Batista, M. (2019a). Student's attention: The use of Brain Waves Sensors in Interactive Videos. International Journal of Advanced Engineering Research and Science, 6(4), 155157.

Bos, A.; Prestes, L.; Pizzato M.; Zaro M. (2019b). Vídeos Educativos:Investigação da Atenção e o Mapeamento do Aprendizado Ativo. In: Abordagens teóricas e reflexões sobre a educação presencial a distância e corporativa. Maringá: Uniedusul Editora.

Bos et al.,(2019). Implementation and analysis of an Intelligent Conversational: Use in a 3D Virtual Museum In A. J. Osório, M. J. Gomes, \& A. L. Valente (Eds.), Challenges 2019: Desafios da Inteligência Artificial, Artificial Intelligence Challenges (1. . ed., pp. 255-260). Braga, Portugal: Universidade do Minho. Centro de Competência.

Herpich, Fabrício; Bos, Andreia; Kuhn, Igor; Guarese, Renan Luigi Martins;Taro uco, Liane Margarida Rockenbach; Wives, Leandro; Zaro, Milton Antônio. "Atividade cerebral no uso de recursos educacionais em realidade aumentada: uma análise da atenção do aprendiz In: XXIX Simpósio Brasileiro de Informática na Educação (Brazilian Symposium on Computers in Education), 2018, Fortaleza. org.crossref.xschema._1.Title@772293c4.,2018.p.1858.

Mulligan, N. W. (1998). The Role of Attention during Encoding in Implicit and Explicit Memory. Journal of Experimental Psychology, 24(1), 27-47.

Neurosky Mindwave Mobile. (2019). Home page. Disponível em: http://store.neurosky.com/products/mindwave-mobile\&amp;gt;. Acesso em julho 2019 